\title{
ANÁLISE DO EFEITO DE DIFERENTES COMBUSTÍVEIS NA CONDIÇÃO DE SMALL EM ALTOS-FORNOS*
}

\author{
Beatriz Fausta Gandra ${ }^{1}$ \\ Henriquison Magela Bottrel Reis ${ }^{2}$ \\ Mauro Vivaldino Fernandes ${ }^{3}$
}

\section{Resumo}

A chave para a operação eficaz de altos-fornos está em elevada produtividade, baixo consumo de combustível e maior liberdade na seleção de matérias-primas. Neste contexto, foram realizados testes de caracterização e comportamento a alta temperatura de diferentes combustíveis para verificar sua potencial utilização sob a forma de small nos altos-fornos. Foi verificada, em escala piloto, melhoria considerável dos resultados para todos os combustíveis analisados em relação à condição de $100 \%$ de sínter na carga metálica. O uso de $5 \%$ de small indica considerável ganho em termos de permeabilidade e redutibilidade, com limite superior em $10 \%$ de small para uso em altos-fornos. Modo geral, o carvão vegetal foi o material de melhor desempenho, seguido pelo coque e coque verde de petróleo. O antracito foi o combustível de pior desempenho sob a forma de small dentre os avaliados. Em relação ao modo de carregamento, é evidente a necessidade de se distribuir o small em meio à carga metálica para se absorver ao máximo seu potencial de melhoria da permeabilidade e da redutibilidade do leito no interior do alto-forno.

Palavras-chave: Small; Alto-Forno; Redução.

\begin{abstract}
EFFECT OF DIFFERENT FUEL AS SMALL IN BLAST FURNACES
Abstract

The blast furnaces effective operations key is high productivity, low fuel rate and greater freedom in raw materials selection. In this context, differents fuels were tested at high temperature in order to verify their potential use in the form of small in blast furnaces. The results, in pilot scale, indicate considerable improvement for all fuels analyzed in relation to the $100 \%$ of sinter condition's. $5 \%$ of small indicates considerable gain in terms of permeability and reducibility, with upper limit at $10 \%$ of small for use in blast furnaces. Overall, the charcoal was the best performance material, followed by coke and pet coke. The antracite had the worse performance in the form of small from those evaluated. Regarding the charging mode, it is clear the importance of the small distribution in the metallic charge in order to improving its permeability and reducibility.
\end{abstract}

Keywords: Small; Blast furnace; Reduction.

1 Membro da ABM; Engenheira Metalurgista, M. Sc., Pesquisadora, Centro de Tecnologia Usiminas, Ipatinga, MG, Brasil.

2 Membro da ABM; Engenheiro Metalurgista, M. Sc., Pesquisador, Centro de Tecnologia Usiminas, Ipatinga, MG, Brasil.

3 Membro da ABM; Engenheiro Metalurgista, M. Sc., Gerente, Gerência Técnica de Redução, Ipatinga, MG, Brasil. 


\section{INTRODUÇÃO}

O propósito do alto-forno é reduzir quimicamente e converter fisicamente o óxido de ferro em gusa líquido. Ele é operado continuamente baseado no princípio de fluxo em contracorrente. No topo são carregados o coque e a carga metálica (sínter, pelota e granulado) em diferentes camadas (Figura 1), que descem por gravidade até a região inferior do forno (cadinho) onde se tornam o produto final (gusa) e escória. Dependendo das condições da carga, este processo requer de cinco a sete horas [1].

O coque impacta fortemente a estabilidade da marcha do alto-forno, afetando diretamente a permeabilidade do leito da carga aos gases e líquidos, podendo contribuir para a produtividade e consumo total de redutores. Além disso, ao longo das últimas décadas, o coque tem se tornado o componente de maior impacto no custo final de produção de ferro gusa nas usinas integradas [2].

Uma das formas de se tentar minimizar este impacto é o uso adequado do small coke (Figura 2), considerando, evidentemente, o equilíbrio de cada usina siderúrgica. Ao ser distribuído junto à carga metálica, este material funciona como fonte de regeneração do gás, melhorando a redução do óxido de ferro. Todavia, para uso do small coke deve ser considerada a possibilidade de sua interferência na permeabilidade da carga do forno e prever ações corretivas, tais como: (i) ajuste na distribuição de carga; (ii) alteração na base de coque; e, (iii) alteração no posicionamento do small coke dentro da sequência de descarga dos materiais.

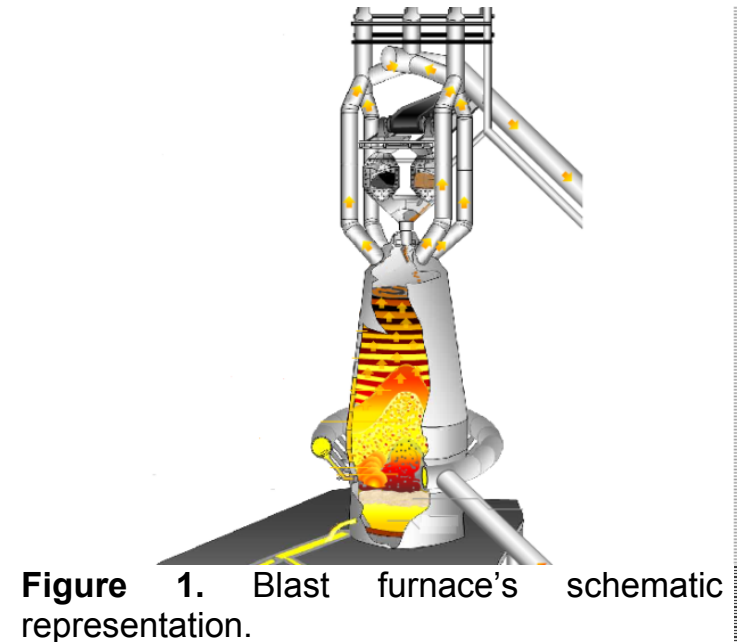

representation.

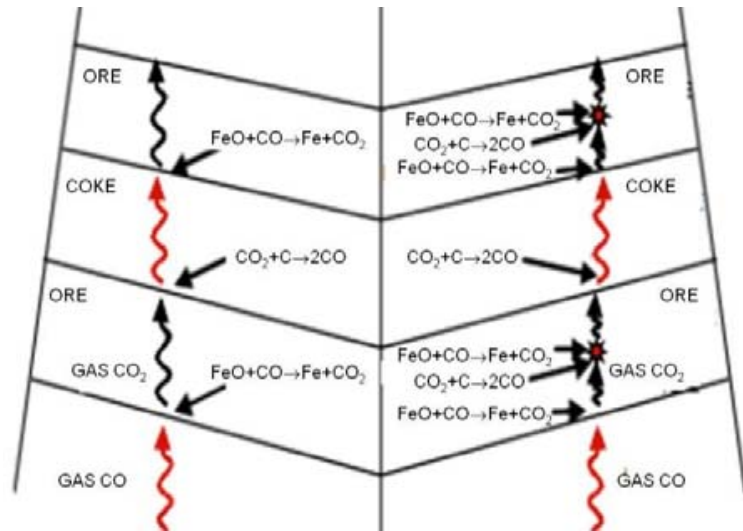

(a) without small coke

(b) with small coke

Figure 2. Schematic representation of the small coke effect in the burden [3].

Segundo Song [4] a mistura de coque na camada de carga metálica é uma maneira econômica de utilização do coque. Condição esta que afeta o comportamento de amolecimento e fusão, a cinética de redução e a permeabilidade do leito, além de permitir o uso completo do coque. No entanto, o mecanismo de atuação do small coke não está bem definido, fato que ainda limita seu uso industrialmente.

No Brasil, o recorde em uso de small coke foi atingido em 2007 pela ArcelorMittal Monlevade [2], cuja estabilidade do processo no alto-forno foi alcançada, em grande parte, em função da seguinte filosofia de distribuição de carga: (i) mínimo de $40 \%$ de coque no material próximo à parede; (ii) mínimo de $70 \%$ de coque no material do centro da carga; e, (iii) mínimo de $75 \mathrm{~kg} /$ tgusa de small coke rate. Além disso, o controle da malha de peneiramento de coque é de fundamental importância para o balanço de coque na usina e para o processo. 
Com a alta participação de small coke na carga, pode-se dizer que o coque do centro é mais preservado no interior do forno, garantindo assim, uma adequada permeabilidade da coluna de carga, refletindo em redução do coke rate e aumento de produtividade [2].

$\mathrm{Na}$ Figura 3 é apresentada a microestrutura do sínter após processo de redução, que pode explicar o fenômeno denominado reduction retardation devido à formação de uma densa casca de ferro metálico, impedindo a passagem dos gases. Sob algumas condições, o mecanismo de controle da redução é principalmente pela difusão no estado sólido, com consequente baixa velocidade. Por outro lado, com a presença de small coke (alta porosidade) o mecanismo de controle da redução é principalmente pela reação química interfacial, muito mais rápida que a anterior [5].

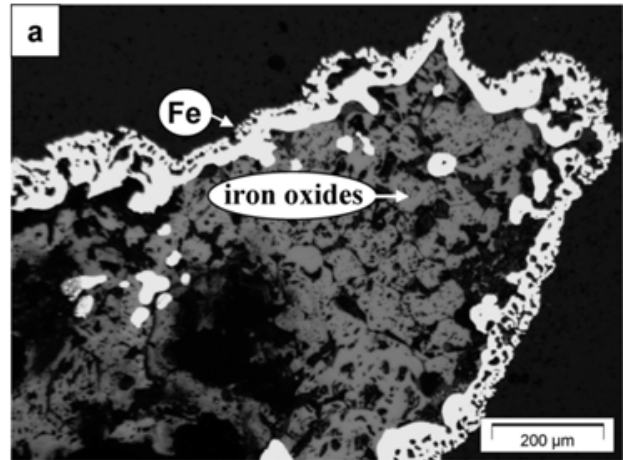

(a) without small coke

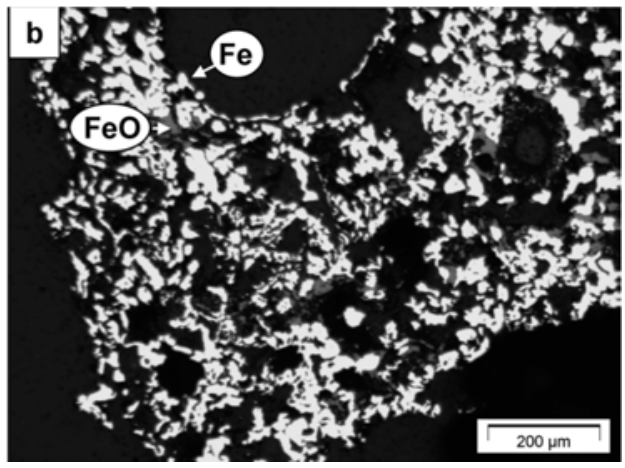

(b) with small coke

Figure 3. Schematic representation of the small coke effect in the burden's reduction [5].

Estudos de pesquisa, citados em Watakabe et al.[6], indicam melhoria das propriedades metalúrgicas da carga metálica, com consequente maior estabilidade da operação dos altos-fornos e redução no consumo de combustível. A perda máxima de pressão é consideravelmente menor com o uso de coque misturado à carga e diminui com o aumento da taxa de small, Figura 4 (a). Os efeitos do coque e da razão de sínter na pressão máxima são apresentados na Figura 4 (b). Em todas as faixas, a queda de pressão é menor quando se tem aumento do uso de coque. Entretanto, é necessária a avaliação do uso deste material, com foco na manutenção da permeabilidade ao gás, além de possível segregação durante o carregamento no alto-forno, devido às diferenças no diâmetro e na densidade do minério e do coque, o que pode dificultar o controle da distribuição de carga.

Atualmente, tem-se discutido de maneira intensa a possibilidade de diminuição do consumo de carbono no alto-forno pela alteração no ponto de equilíbrio para a transição FeO-Fe (W para $\mathrm{W}^{1}$, Figura 5) afetando a redução da temperatura da zona de reserva térmica.

A ideia está derivada do diagrama de RIST indicando que uma redução na temperatura de equilíbrio entre o gás redutor e a wustita poderia alterar a composição do gás redutor de maneira a se obter menor $\mathrm{CO}$ e maior teor de $\mathrm{CO}_{2}$. Isto aumentaria a eficiência de utilização do gás, resultando em menor consumo de agente redutor [7]. Uma maneira de se obter essa situação seria com o uso de combustível mais reativo, que levou a estudos para avaliar inclusive o efeito do char e da cinza do carvão no comportamento do coque. 


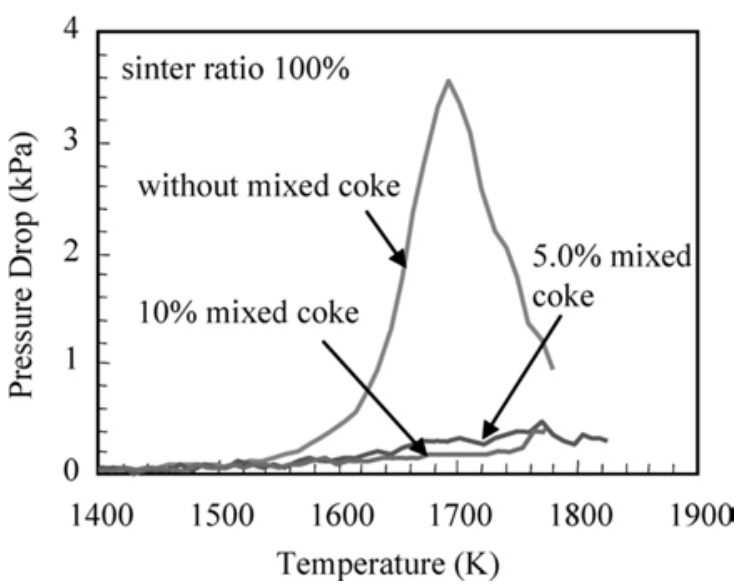

(a) $100 \%$ sinter in the burden

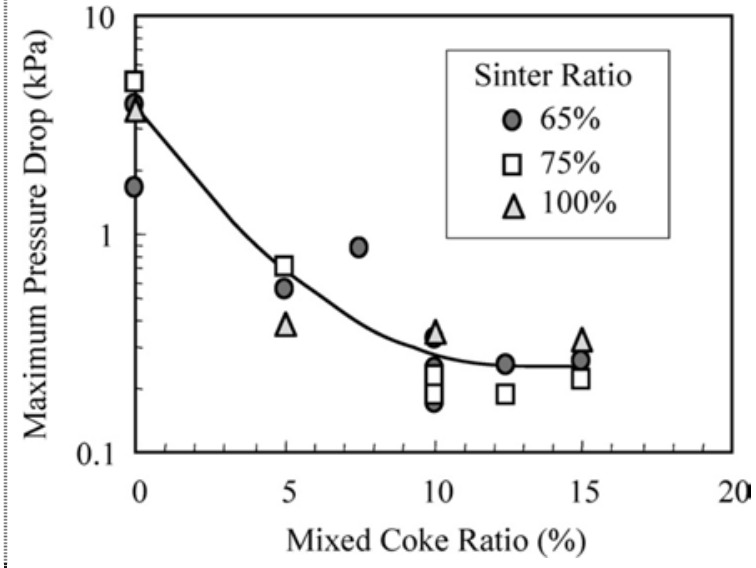

(b) sinter ratio (NPO balance)

Figure 4. Effect of proportion of coke mixed with metallic burden in gas permeability in the high temperature region [6].

Na Figura 6 é apresentado um levantamento da quantidade de small utilizada em fornos europeus e norte-americanos no ano de 2013. Em destaque, dados dos fornos da Usiminas Ipatinga.

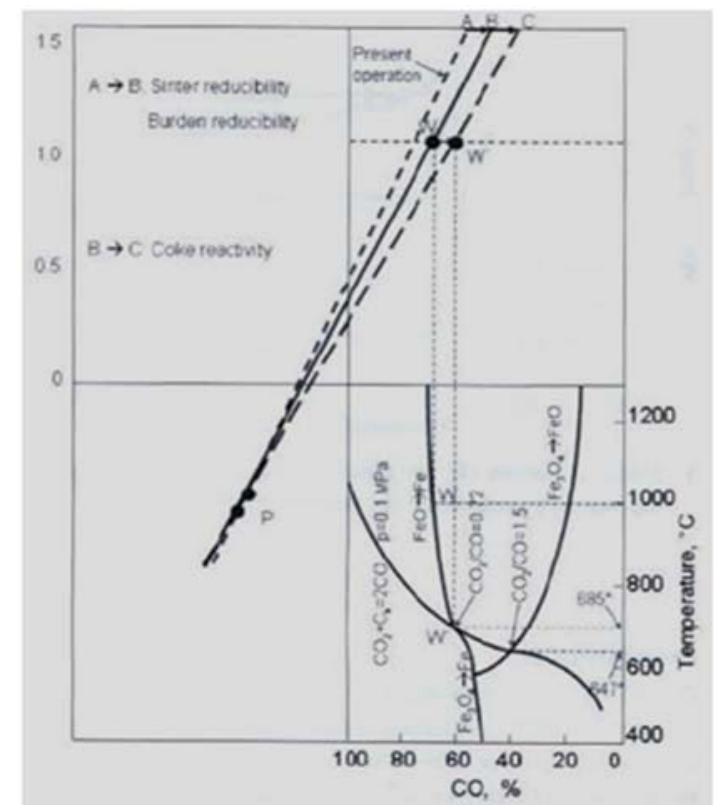

Figure 5. Operation Diagram RIST combined with Baur-Glaessner equilibrium diagram and Boudouard reaction $\mathrm{P}=0,1 \mathrm{MPa}$ [7].

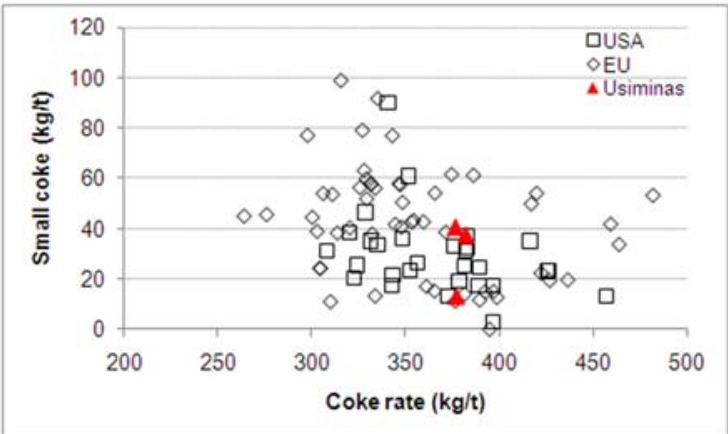

Figure 6. Small rate used in Europe, USA and Usiminas Ipatinga, 2013.

A grande maioria das usinas utilizam small coke na faixa de $20 \mathrm{~kg} / \mathrm{t}$ a $60 \mathrm{~kg} / \mathrm{t}$, com algumas poucas entre $80 \mathrm{~kg} / \mathrm{t} \mathrm{e} 100 \mathrm{~kg} / \mathrm{t}$. Observa-se uma ligeira tendência de queda do coke rate para fornos com maior consumo de small e a possibilidade de aumento do uso de small por parte da Usiminas, especialmente no Alto-Forno 3, que apresentou média em 2013 igual a 13,2 kg/t. 


\section{MATERIAIS E MÉTODOS}

Baseado na bibliografia consultada e na disponibilidade de materiais no mercado, optou-se por avaliar os seguintes materiais: coque (CQ); carvão vegetal (CV); coque verde de petróleo (CVP); e, antracito (AT). Os testes foram realizados considerando três faixas de granulometria: (5 a 10) mm, (10 a 15) $\mathrm{mm}$ e $(15$ a 20) $\mathrm{mm}$; e, o percentual em massa dos combustíveis variando em $0 \% ; 5 \%$ e $10 \%$ do sínter a ser utilizado no teste de amolecimento e fusão (A\&F). A carga metálica utilizada foi $100 \%$ sínter, amostrado industrialmente, com granulometria entre $10 \mathrm{~mm}$ e $15 \mathrm{~mm}$. A caracterização dos materiais foi feita conforme descrição a seguir.

Análise química completa, realizada em equipamentos de fluorescência de raios$X$, espectrometria de plasma e, também, por via úmida;

Porosimetria por intrusão de mercúrio, na qual se medem poros de diâmetros entre $0,005 \mu \mathrm{m}$ e $360 \mu \mathrm{m}$. A amostra é seca em estufa a $150{ }^{\circ} \mathrm{C}$ por 1 hora. $\mathrm{O}$ ensaio é validado caso seja utilizado entre $25 \%$ e $90 \%$ do mercúrio contido na haste.

Caracterização petrográfica, realizada por meio de microscopia óptica. Foi utilizada luz polarizada e normal com intensidade de iluminação fixada em torno de 10 volts, através das objetivas de aumento de 10x, 20x, 50x e 100x.

Análise termogravimétrica (TGA), com taxa de aquecimento igual a $10^{\circ} \mathrm{C} / \mathrm{min}$, em ambiente oxidante (ar) até a temperatura de $1500^{\circ} \mathrm{C}$.

Ensaios a alta temperatura, realizados no forno de amolecimento e fusão (FA\&F). Para tanto, numa primeira etapa o carregamento do material sínter/small foi feito de maneira homogênea; numa segunda fase, foram testados os materiais combustíveis numa granulometria igual a $3 \mathrm{~mm}$, concentrado em uma camada de $10 \mathrm{~mm}$ no meio de duas camadas de $30 \mathrm{~mm}$ do sínter (Figura 7).

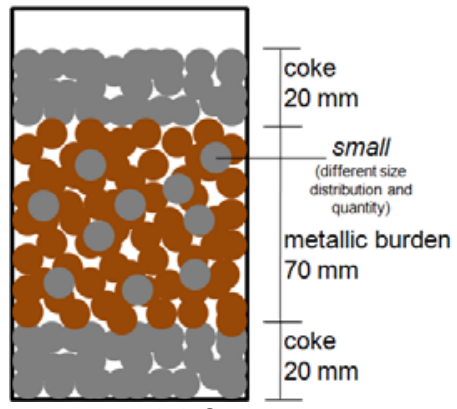

(a) first step

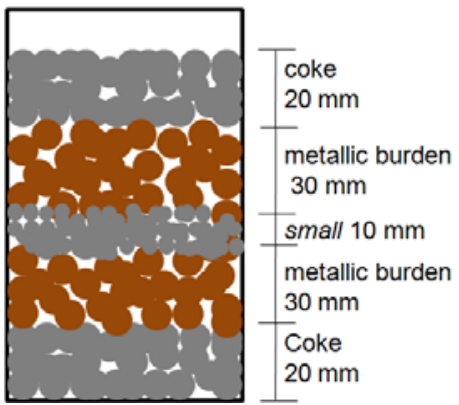

(b) second step

Figure 7. Crucible's schematic representation for S\&M test.

Os principais parâmetros obtidos pelos ensaios de amolecimento e fusão são:

- Ts - temperatura de início da zona coesiva, que correspondente ao instante em que a queda de pressão atinge $200 \mathrm{mmH}_{2} \mathrm{O}$ durante o aquecimento da amostra;

- $\Delta \mathbf{T}$ - estimativa da espessura da zona coesiva, corresponde ao aumento de temperatura experimentado pela carga metálica na região de queda de pressão mais significativa do alto-forno;

- $\Delta$ Pmax - queda máxima de pressão, mede a perda de carga no leito;

- S - área sob o pico de perda de pressão do gás, que é um indicador da permeabilidade global do leito durante o ensaio; e,

- RTs e R1200 - grau de redução em altas temperaturas. 


\section{RESULTADOS E DISCUSSÃO}

\subsection{Caracterização dos Materiais}

$\mathrm{Na}$ Tabela 1 são apresentados os resultados da análise química dos combustíveis. Destaque para o baixo carbono fixo do CV $(74,04 \%)$, que reflete em baixo poder caloríico (6891 cal/g); por outro lado, apresentou baixíssimo teor de enxofre $(0,2 \%)$. Já o CVP se destaca pelo baixo teor de cinza $(0,12 \%)$, que o classifica com o mais elevado poder calorífico $(8705 \mathrm{cal} / \mathrm{g})$ dentre os combustíveis analisados.

Table 1. Fuels's chemical analysis

\begin{tabular}{|c|c|c|c|c|c|}
\hline & Parâmetro & $\mathbf{C Q}$ & CV & CVP & AT \\
\hline \multirow{3}{*}{ Immediate analysis (\%) } & Gray & 9,76 & 2,06 & 0,12 & 6,15 \\
\hline & Volatile Matter & 0,85 & 23,90 & 12,10 & 4,33 \\
\hline & Carbon Fixed & 89,39 & 74,04 & 87,78 & 89,52 \\
\hline \multirow{5}{*}{ Elementary analysis (\%) } & C & 85,2 & 77,1 & 90,3 & 85,5 \\
\hline & $\mathrm{H}$ & n.a.* & 4,41 & n.a. & n.a. \\
\hline & $\mathrm{O}$ & 0,53 & 15,00 & 1,05 & 3,74 \\
\hline & $\mathrm{N}$ & n.a. & 0,39 & n.a. & n.a. \\
\hline & S & 0,70 & 0,20 & 0,75 & 0,91 \\
\hline \multirow{5}{*}{ Gray composition (\%) } & $\mathrm{SiO}_{2}$ & 46,6 & 7,5 & 18,7 & 42,4 \\
\hline & $\mathrm{Al}_{2} \mathrm{O}_{3}$ & 27,50 & 4,01 & 5,34 & 17,60 \\
\hline & $\mathrm{Fe}_{2} \mathrm{O}_{3}$ & 12,8 & 8,7 & 15,0 & 26,6 \\
\hline & $\mathrm{CaO}$ & 4,03 & 36,39 & 3,50 & 4,23 \\
\hline & $\mathrm{MgO}$ & 1,15 & 19,10 & 2,57 & 1,99 \\
\hline \multicolumn{2}{|c|}{ Higher Calorific Value (cal/g) } & 7032 & 6891 & 8705 & 7344 \\
\hline
\end{tabular}

A porosimetria foi obtida para cada uma das três faixas de combustível utilizado nos testes de A\&F (Figura 8). No entanto, não se observa grandes variações desta propriedade entre as faixas granulométricas.

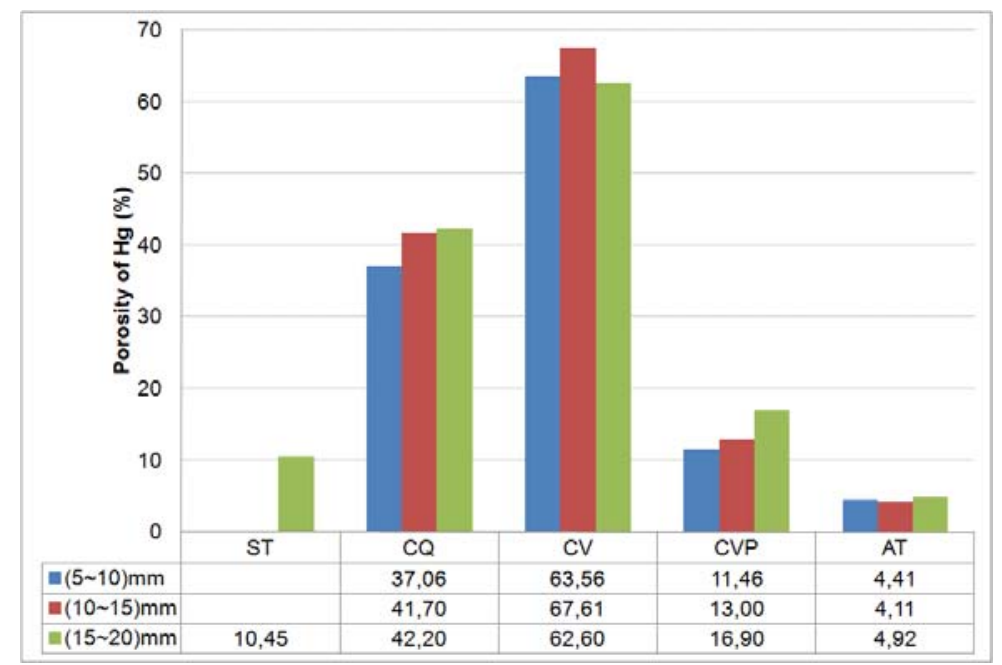

Figure 8. Pore-size distribution results.

Dentre os combustíveis analisados, o CV apresentou a maior porosidade $(>60 \%)$, seguido pelo CQ ( 40\%), CVP ( 15\%), e, em último lugar, o AT $(<5 \%)$, com porosidade bastante baixa, resultados confirmados pela petrografia (Figura 9 ). 

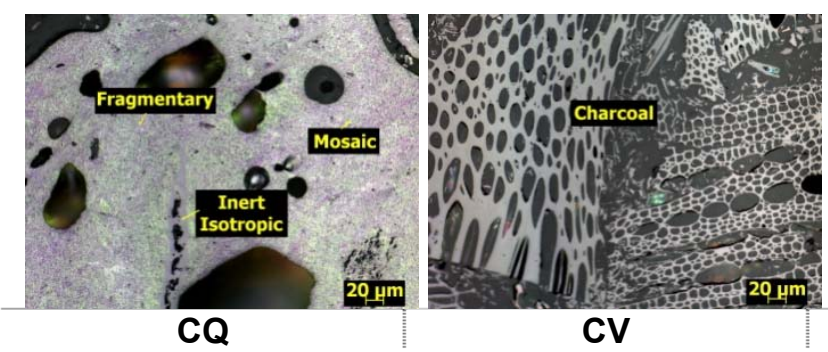

CV

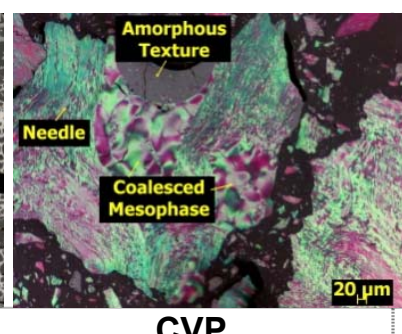

CVP

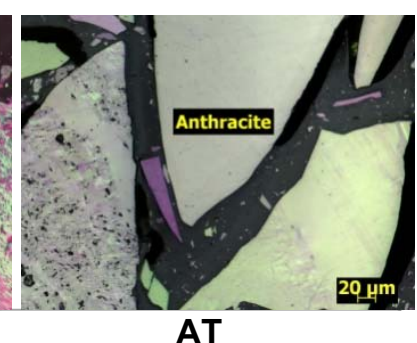

Figure 9. Photomicrograph of constituents.

Pela análise termogravimétrica (Figura 10), tem-se que o CV é o material que reage a temperaturas mais baixas, seguida pelo CVP, AT e, em último lugar, o CQ. Este comportamento deve estar associado à sua quantidade de volátil.

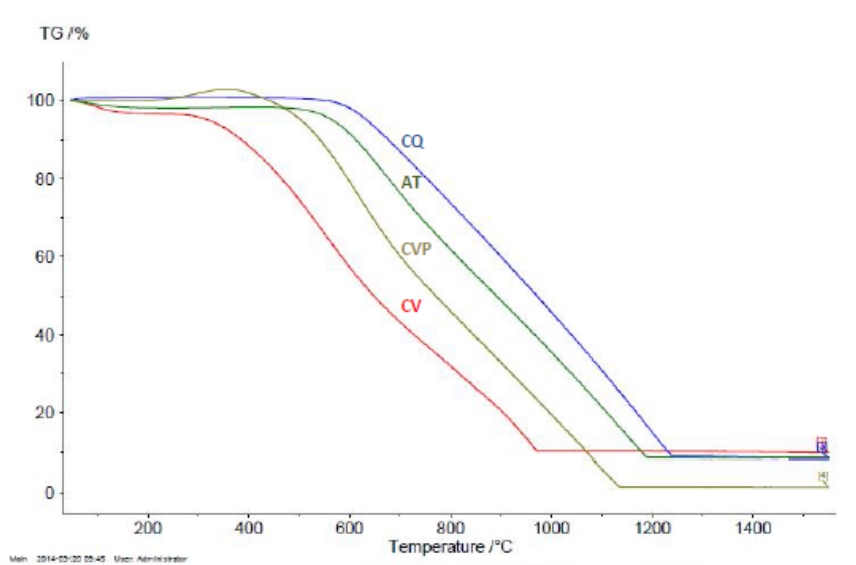

Figure 10. Thermogravimetric analysis.

\subsection{Ensaios a Alta Temperatura}

Nas Figuras 11 e 12 pode-se observar graficamente o efeito dos combustíveis na permeabilidade do leito.

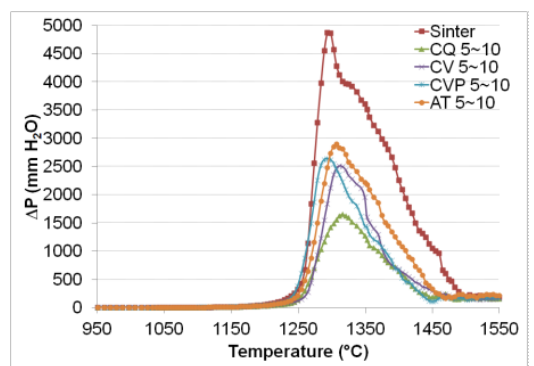

(a) $5 \mathrm{~mm}$ a $10 \mathrm{~mm}$

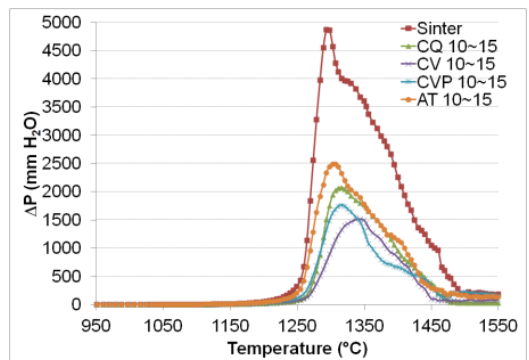

(b) $10 \mathrm{~mm}$ a $15 \mathrm{~mm}$

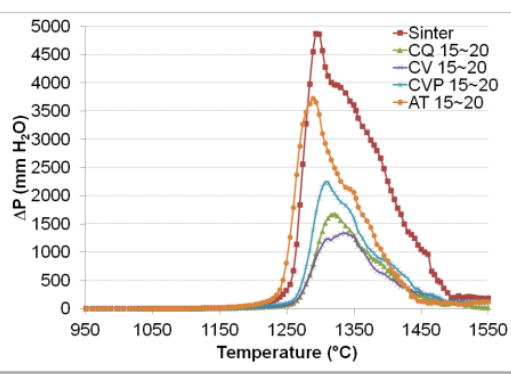

(c) $15 \mathrm{~mm}$ a $20 \mathrm{~mm}$

Figure 11. Differential pressure results versus temperature, fuel $5 \%$.

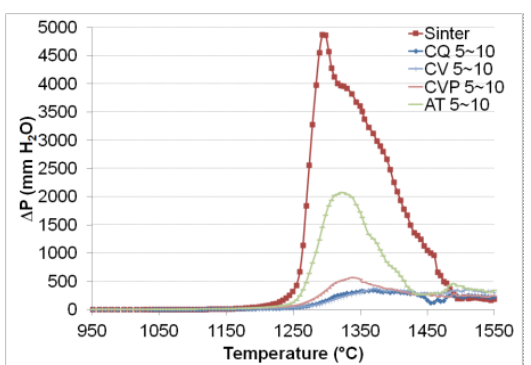

(a) $5 \mathrm{~mm}$ a $10 \mathrm{~mm}$

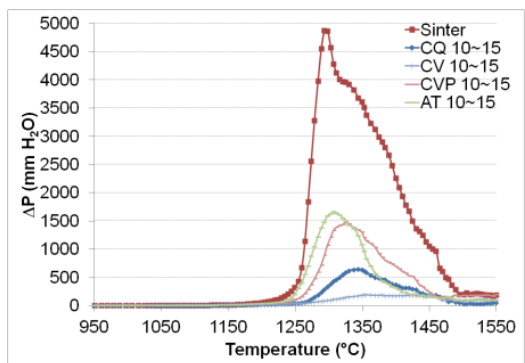

(b) $10 \mathrm{~mm}$ a $15 \mathrm{~mm}$

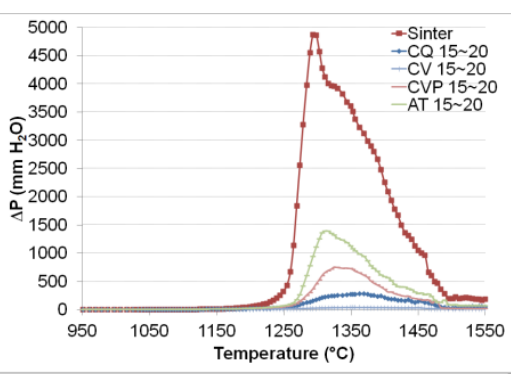

(c) $15 \mathrm{~mm}$ a $20 \mathrm{~mm}$

Figure 12. Differential pressure results versus temperature, fuel $10 \%$. 
Para todos os combustíveis, o valor de $S$ é consideravelmente inferior àquela condição quando se tem $100 \%$ sínter na carga. O CQ apresenta excelente resultado quando utilizado em granulometria inferior à da carga metálica. O CV e o CVP apresentam melhor resultado quando utilizados em granulomentria similar ou superior àquela da carga metálica. O AT apresentou o pior resultado em todas as situações. Estes resultados estão sumarizados na Figura 13, pela qual fica facilitada a análise comparativa.

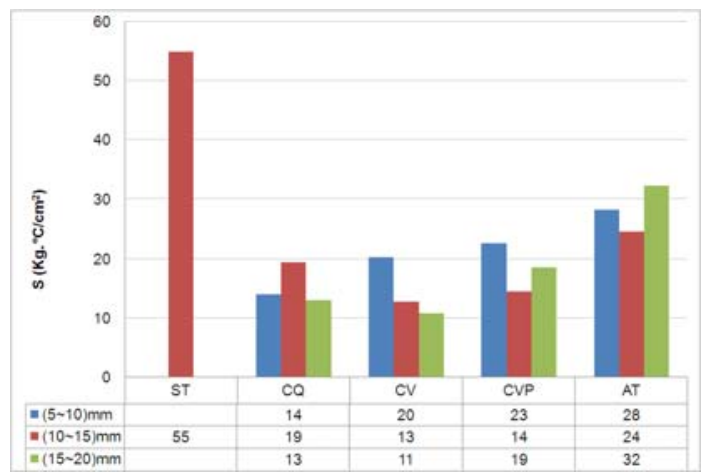

(a) $5 \%$ of small

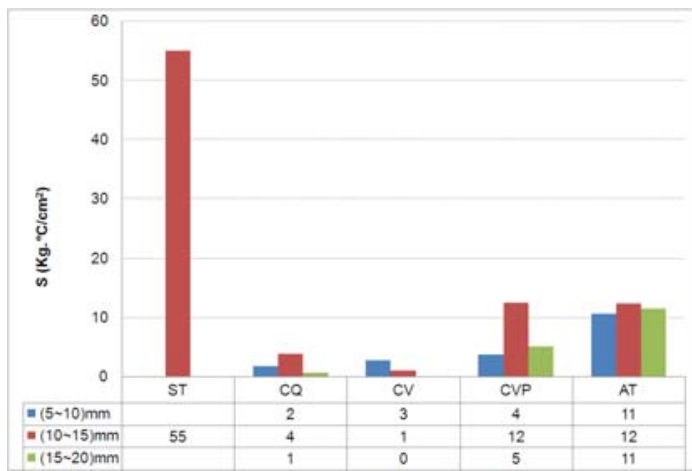

(b) $10 \%$ of small

Figure 13. Area under the pressure curve (S).

A temperatura de início de amolecimento teve maior relação com o percentual de combustível sob a forma de small, conforme é apresentado na Figura 14. Este fato pode estar associado a uma melhor redutibilidade da carga metálica.

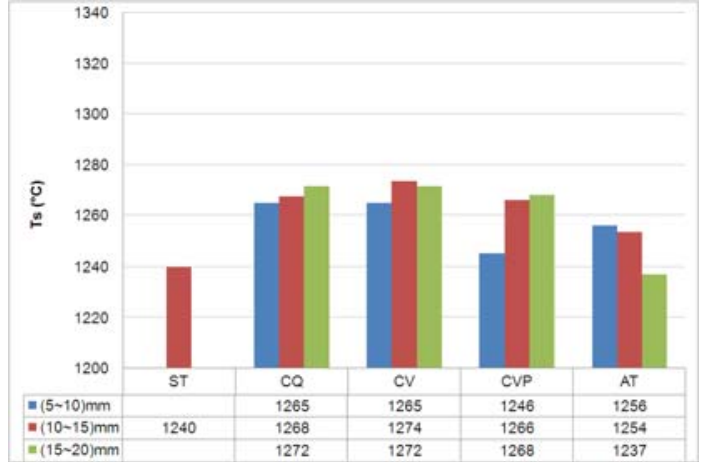

(a) $5 \%$ of small

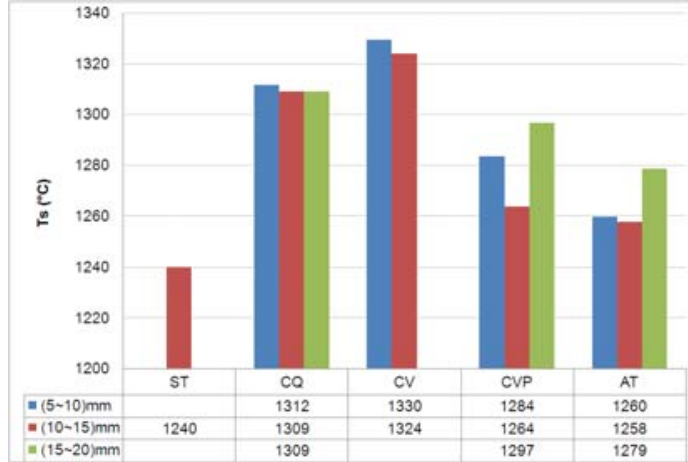

(b) $10 \%$ of small

Figure 14. Softening temperature.

Pela Figura 15 ressalta-se o efeito do CV na redutibilidade da carga metálica.

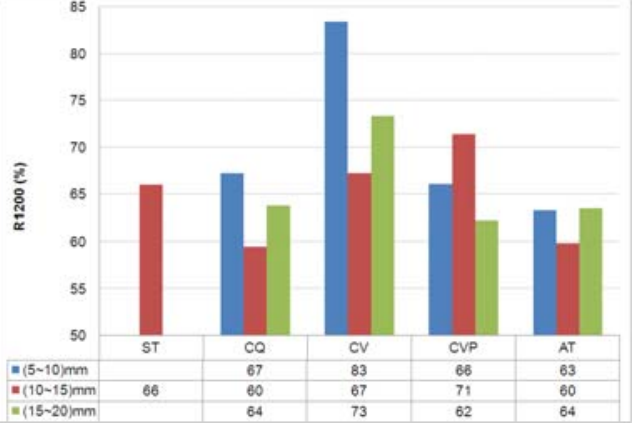

(a) $5 \%$ of small

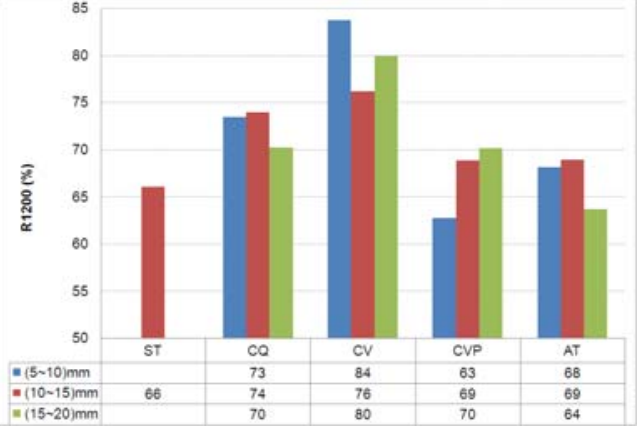

(b) $10 \%$ of small

Figure 15. Reducibility at $1200^{\circ} \mathrm{C}$. 
A segunda etapa de testes, na qual o combustível ficou concentrado no meio de duas camadas de sínter de $30 \mathrm{~mm}$ (Figuras 16 e 17), deixa evidente a necessidade de se distribuir o small em meio à carga metálica no sentido de se absorver ao máximo seu potencial de melhoria da permeabilidade e da redutibilidade do leito no interior do alto-forno.

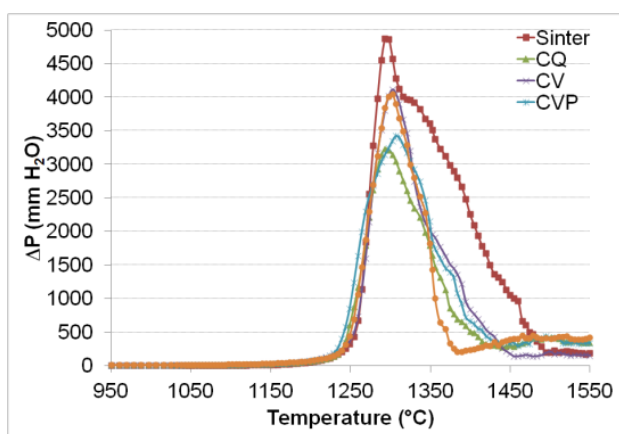

Figure 16. Differential pressure versus temperature.

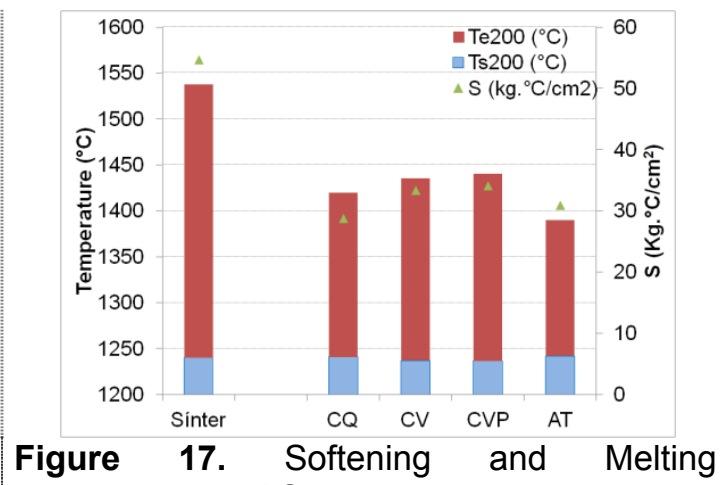

temperature and $\mathrm{S}$.

\subsection{Análise Estatística}

As principais análises estatísticas são apresentadas na Figura 18.

Em relação à temperatura de início de amolecimento (Figura 18 (a)) se observa clara elevação quando comparada ao sínter $\left(1240^{\circ} \mathrm{C}\right)$, com maior efeito para o $\mathrm{CV}$, seguido pelo $C Q$ e CVP, sendo que a granulometria mais grosseira reflete em maior temperatura de início de amolecimento. $\mathrm{Na}$ prática, isto indica melhor permeabilidade ao forno, que opera com zona coesiva mais próxima ao cadinho. $\mathrm{O}$ AT foi o material de menor efeito neste parâmetro, independente da granulometria. Associado a este fenômeno, a redução da temperatura final de fusão tem como consequência menor espessura da zona coesiva (Figura 18 (b)), que apresenta considerável melhora em relação ao sínter sozinho $\left(298^{\circ} \mathrm{C}\right)$. Neste aspecto, pode-se afirmar melhor efeito para o coque e o carvão vegetal.

Em termos de máxima queda de pressão (Figura 18 (c)) o CV se apresenta bem similar ao CQ. O AT apresenta o pior desempenho, seguido pelo CVP, ainda assim, resultado consideravelmente superior ao sínter sozinho $\left(5000 \mathrm{mmH}_{2} \mathrm{O}\right)$. Neste caso, granulometria mais grosseira indica melhor resultado, à exceção do AT que apresentou o melhor resultado quando usado entre $10 \mathrm{~mm}$ e $15 \mathrm{~mm}$.

Os resultados de carbono solution loss e R1200 (Figuras 18 (d), e 18 (e)) indicam melhor redutibilidade da carga com o uso do carvão vegetal, com consequente redução no consumo de combustível. Neste caso, a granulometria teve pouca influência para o CQ e o AT; intermediária para o CVP; e, elevada para o CV. Este combustível apresentou melhor resultado em granulometria mais fina $(5 \mathrm{~mm}$ a $10 \mathrm{~mm}$ ), diferente do CVP que apresenta melhor resultado para granulometria similar à carga metálica $(10 \mathrm{~mm}$ a $15 \mathrm{~mm})$ utilizada no teste A\&F.

Baseado nas características analisadas pode-se afirmar que o carvão vegetal foi o material de melhor desempenho, seguido pelo coque e coque verde de petróleo. $\mathrm{O}$ antracito foi o combustível de pior desempenho sob a forma de small dentre os avaliados. 
Means and 95,0 Percent LSD Intervals

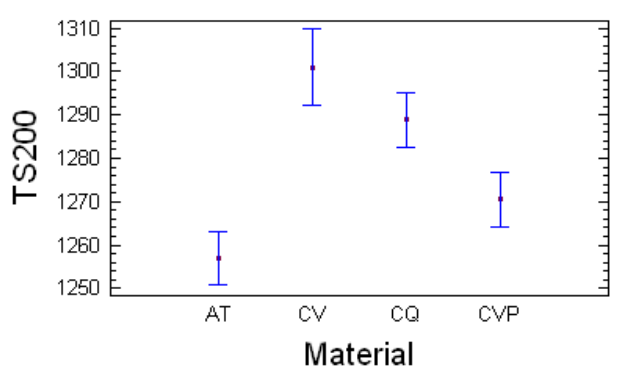

Interaction Plot

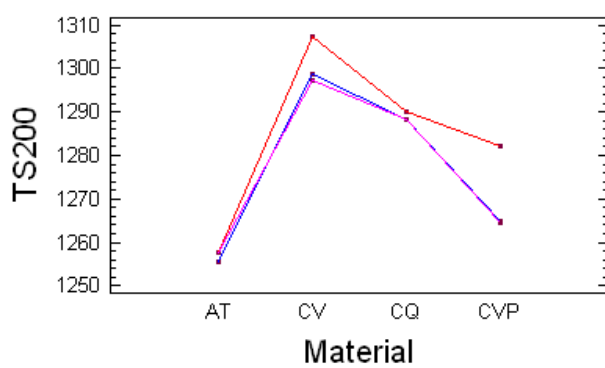

Granulometria - 10 a $15 \mathrm{~mm}$ -5 a $10 \mathrm{~mm}$

(a) effect of the material type/particle size in the softening temperature Means and 95,0 Percent LSD Intervals

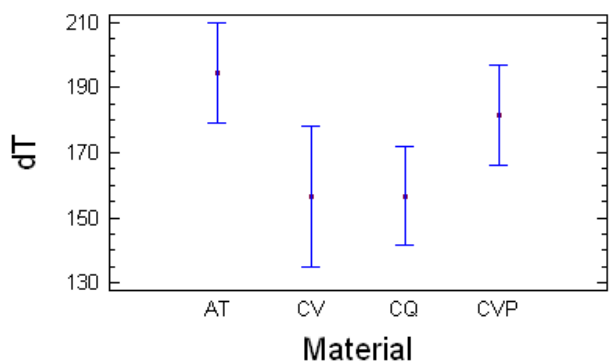

Interaction Plot

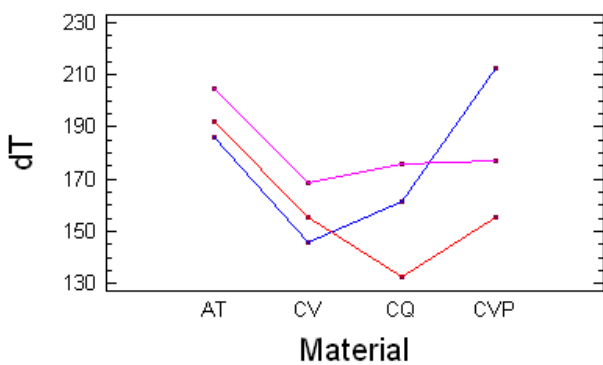

(b) effect of the material type/particle size in the cohesive zone thickness Means and 95,0 Percent LSD Intervals

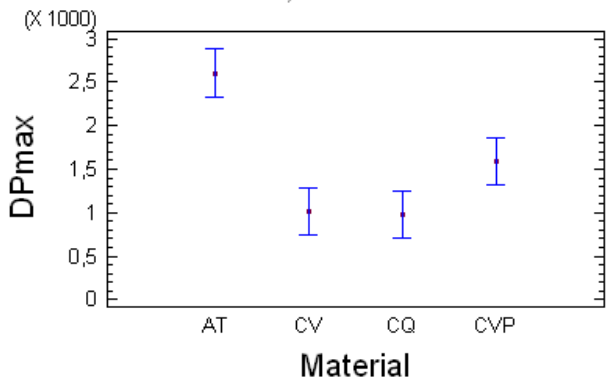
Interaction Plot

(c) effect of the material type/particle size at maximum pressure Means and 95,0 Percent LSD Intervals

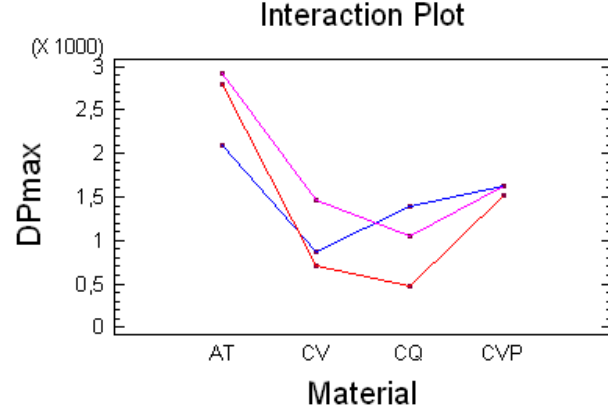

Granulometria -10 a $15 \mathrm{~mm}$ -15 a $20 \mathrm{~mm}$ -5 a $10 \mathrm{~mm}$

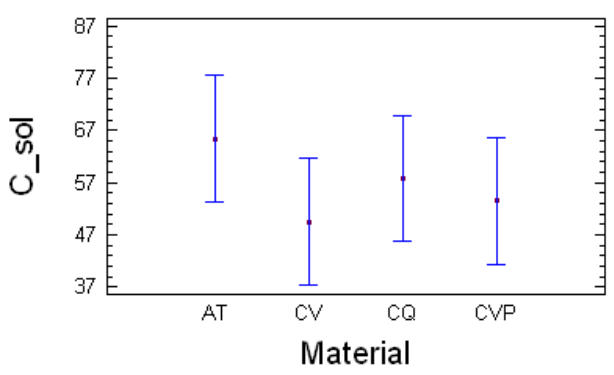
Interaction Plot

(d) effect of the material type/particle size in carbon solution loss Means and 95,0 Percent LSD Intervals
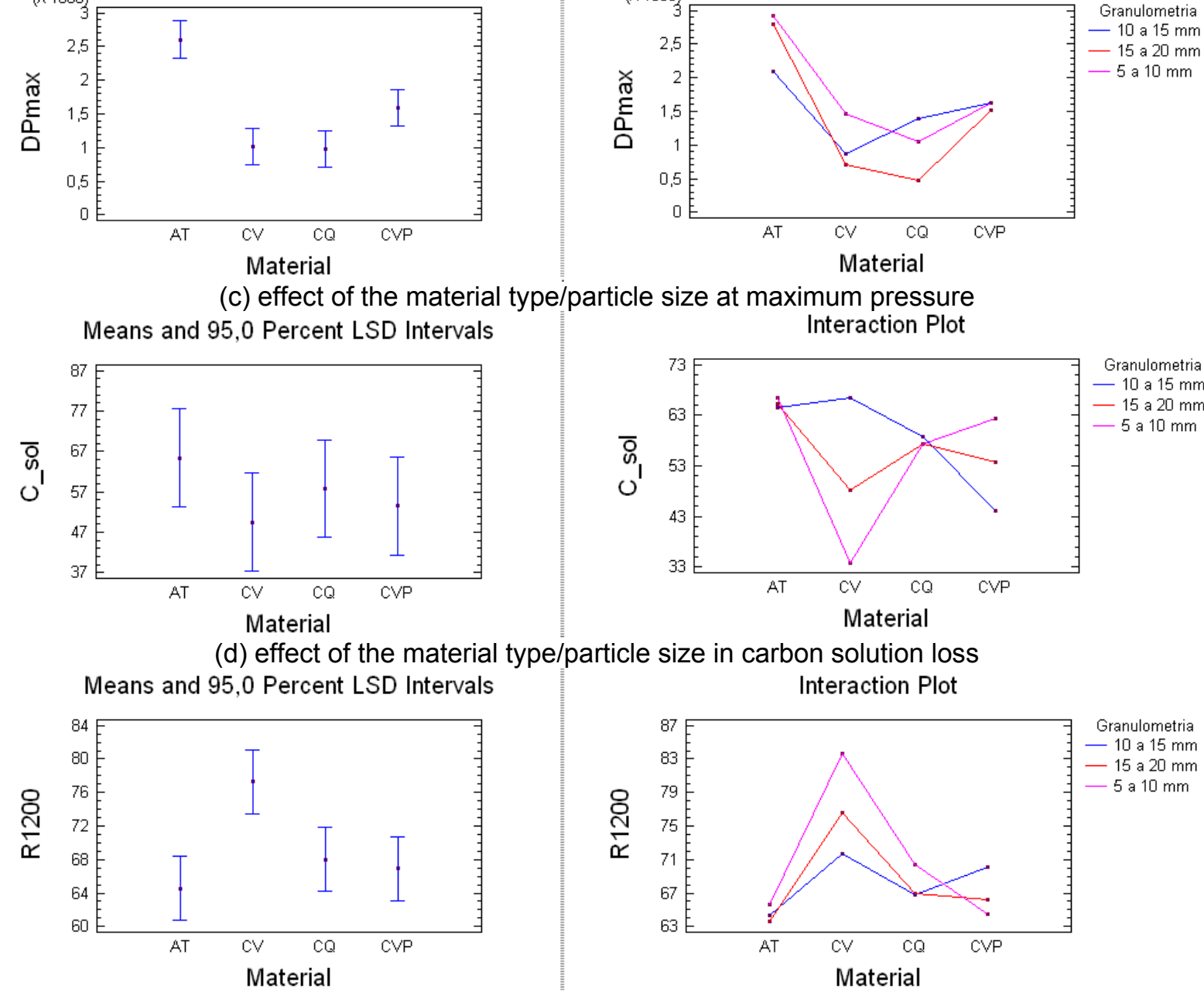

Granulometria -10 a $15 \mathrm{~mm}$ -15 a $20 \mathrm{~mm}$

(e) effect of the material type/particle size in reducibility at $1200^{\circ} \mathrm{C}$

Figure 18. Statistical analysis of the S\&M results. 


\section{CONCLUSÃO}

Dentre os combustíveis avaliados, o carvão vegetal se destaca pelo seu baixíssimo teor de enxofre e o coque verde de petróleo pelo baixo teor de cinza.

Houve melhora considerável dos resultados para todos os combustíveis analisados em relação à condição de $100 \%$ de sínter na carga, sendo $10 \%$ o limite para uso e small em altos-fornos, já que é uma condição na qual se tem máxima permeabilidade da carga. De modo geral, o carvão vegetal foi o material de melhor desempenho, seguido pelo coque e coque verde de petróleo. $O$ antracito foi o combustível de pior desempenho sob a forma de small dentre os avaliados.

Considerando todos os parâmetros analisados, em termos de distribuição de tamanho do small, o carvão vegetal e o coque podem ser aplicados em granulometria superior à carga metálica. O coque verde de petróleo e o antracito apresentaram melhor resultado quando foi usada granulometria similar àquela da carga metálica. Em relação ao modo de carregamento, testes realizados deixaram evidente a necessidade de se distribuir o small em meio à carga metálica no sentido de se absorver ao máximo seu potencial de melhoria da permeabilidade e da redutibilidade do leito no interior do alto-forno.

\section{REFERÊNCIAS}

1 BABICH, A.; SENK, D.; GUDENAU, H. W.; MAVROMMATIS, K. Th. "Ironmaking". Textbook, Institut für Eisenhüttenkunde der RWTH Aachen University, Department of Ferrous Metallurgy, 2008.

2 ANDRADE, F. C.; SANTOS, R. J.; OLIVEIRA, W. B. "Evolução da operação do AltoForno A da ArcelorMittal Monlevade com Elevada Participação de Small Coke na Carga Redutora". ABM, $39^{\circ}$ Seminário de Redução de Minério de Ferro e Matérias-primas e $10^{\circ}$ Seminário Brasileiro de Minério de Ferro, Ouro Preto - MG, 2009.

3 FUJIHARA, F. K.; GUSHIKEN, J. I.; DEFENDI, G. A.; PERDIGÃO, L. P. "Coke Consuption Philosophy at ArcelorMittal Tubarão Blast Furnaces". $6^{\text {th }}$ IAS Reduction Conference, Rosário - Argentina. Novembro, 2007.

4 SONG, Q. "Effect of Nut Coke on the Performance of the Ironmaking Blast Furnace." $\mathrm{PhD}$ Thesis - Department of Materials Science and Engineering of Delft University of Technology (TUDelft). July, 2013.

5 MOUSA, E. A.; BABICH, A.; SENK, D. "Effect of Nut Coke-sinter Mixture on the Blast Furnace Performance". ISIJ International, Vol. 51, No. 3, pp. 350-358, 2011.

6 WATAKABE, S.; TAKEDA, K.; NISHIMURA, H.; GOTO, S.; NISHIMURA, N.; UCHIDA, T.; KIGUCHI, M. "Development of High Ratio Coke Mixed Charging Technique to the Blast Furnace". ISIJ International, Vol. 46, No.4, pp. 513-522, 2006.

7 BABICH, A.; SENK, D.; GUDENAU, H. W. "Effect of coke reactivity and nut coke on blast furnace operation". Ironmaking and Steelmaking, Vol. 36, No 3, pp. 222 a 229. 2009. 